\title{
Associação do tempo de sono com os fatores de risco antropométricos e cardiovasculares em universitários
}

\author{
Association of sleep time with anthropometric and cardiovascular risk factors \\ in university students
}

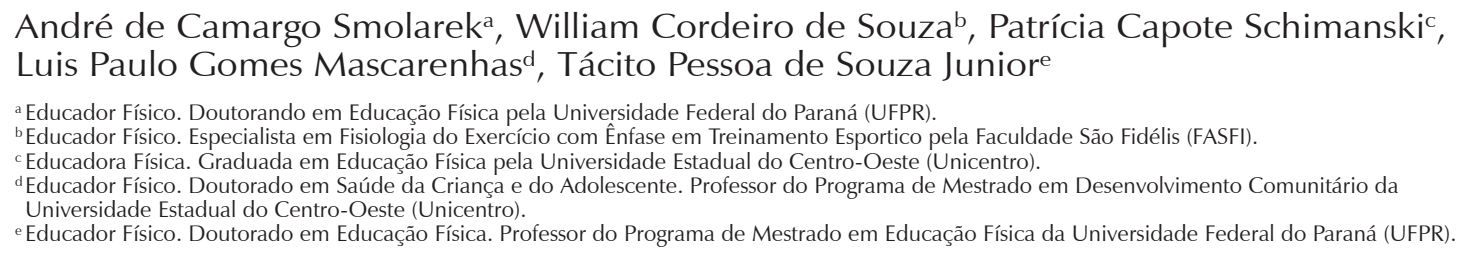

RESUMO

Objetivo: Avaliar a associação entre o tempo de sono com os fatores de riscos a saúde em universitários.

Materiais e Métodos: O presente estudo de característica transversal foi constituído por 202 universitários (126 homens e 76 mulheres), com idades entre 18 e 46 anos. Foram mensuradas as variáveis antropométricas de massa corporal (MS), estatura (E), circunferência cintura (CC), índice de massa corporal (IMC) e relação cintura estatura (RCE). Foram coletados os dados de pressão arterial sistólica (PAS) e diastólica (PAD). A qualidade do sono foi analisada com base no instrumento denominado Índice de Qualidade do Sono de Pittsburgh (PSQI).

Resultados: No grupo de universitários avaliados observou-se que uma média de $7,1 \pm 1,4$ horas de sono apresenta associação benéfica para a manutenção da PAS $(O R=0,31 ; p=0,04)$. Já nas universitárias constatou que uma média $7,6 \pm 1,4$ horas de sono apresenta associação com a RCE para a prevenção dos fatores risco de desenvolvimento de doenças cardiovasculares $(\mathrm{OR}=2,57 ; p=0,03)$.

Conclusão: Sendo assim, conclui-se que 7,1 horas de sono contribui para a manutenção da PAS em universitários do sexo masculino. Enquanto isso, no sexo feminino, 7,6 horas de sono contribui na prevenção de fatores de risco de desenvolvimento de doenças cardiovasculares através da RCE.

Palavras-chave: fatores de risco; pressão arterial; sono.

Objective: To assess the association between sleep time with the health risk factors in university students.

Materials and Methods: The present cross-sectional study included 202 university students (126 males and 76 females), aged between 18 and 46 years. The anthropometric variables of body mass (BM), stature (S), waist circumference (WC), body mass index (BMI) and waist stature ratio (WSR) were measured. To check for possible cardiovascular risks, the systolic (SAP) and diastolic (DAP) blood pressure data were collected. The quality of sleep was analyzed on the basis of the instrument called the Pittsburgh Sleep Quality Index (PSQI).

Results: In the group of male students evaluated it was observed that an average of $7.1 \pm 1.4$ hours of sleep presents beneficial association for the maintenance of SAP $(O R=0.31 ; p=0.04)$. However, in female students, an average of $7.6 \pm 1.4$ hours of sleep presents association with the WSR for the prevention of risk factors for the development of cardiovascular diseases $(O R=2.57$; $p=0.03)$.

Conclusion: Therefore, it is concluded that 7.1 hours of sleep contributes to the maintenance of SAP in male college students. On the other hand, in females, 7.6 hours of sleep contribute to the prevention of risk factors for the development of cardiovascular diseases through WSR.

Keywords: risk factors; blood; sleep. 


\section{INTRODUÇÃO}

O sono e repouso são funções restauradoras necessárias para a preservação da vida e deve fazer parte do cotidiano de todos os seres humanos ${ }^{1}$. O processo de desencadeamento do sono é regulado pelo ritmo circadiano, o qual é influenciado por condições endógenas, sociais e ambientais². Padrões adequados de sono são de extrema relevância para uma boa qualidade de vida, pois auxiliam na consolidação da memória, na termorregulação, na conservação, na restauração da energia e restabelecem o metabolismo energético cerebral ${ }^{3}$.

Atualmente, a privação do sono aparece como um problema cada vez mais frequente da sociedade moderna ${ }^{4}$. A baixa qualidade do sono está associada ao surgimento de doenças crônicas não transmissíveis, como o diabetes mellitus tipo 2, a hipertensão arterial sistêmica e a comorbidades como dislipidemia, litíase biliar e doença hepática ${ }^{5}$. Costa et al. ${ }^{6}$ destacam que grande maioria dos universitários que estudam à noite apresentam alterações e fragmentação na qualidade do sono devido a dupla jornada.

A qualidade de sono está diretamente relacionada ao desempenho cognitivo, à aprendizagem e memória, uma vez que a privação parcial ou total do sono manifesta relação direta com o cansaço e sonolência diurna excessiva, ocasionando elevação do estado de estresse emocional e perda do rendimento das atividades rotineiras. As alterações podem ser oriundas dos horários de trabalho e/ou escolares dos universitários, principalmente nas épocas destinadas às provas acadêmicas, no qual o acúmulo de tarefas reduz o tempo e a qualidade do sono, e assim estimulam algumas alterações hormonais? .

Haja vista ao exposto acima, o estilo de vida dos universitários pode ser comprometido e estar fora dos padrões adequados para uma boa qualidade de vida. O consumo de drogas licitas e o sedentarismo são fatores contribuintes para a perda da qualidade do sono, o que pode acarretar em prejuízos à saúde, promovendo alterações no estado fisiológico e até mesmo no rendimento acadê$\mathrm{mico}^{8}$. Diante do exposto, o presente estudo tem o objetivo avaliar a associação entre o tempo de sono com os fatores de risco antropométricos e cardiovasculares em universitários.

\section{MATERIAIS E MÉTODOS}

Trata-se de um estudo de característica transversal, constituído por 202 acadêmicos do curso de educação física da Faculdade Guairacá, no município de Guarapuava, Paraná, Brasil, entre Fevereiro de 2011 a dezembro de 2012.
Todos os participantes foram previamente informados em relação aos procedimentos aos quais seriam submetidos. Em seguida assinaram um termo de consentimento livre e esclarecido (TCLE). O projeto foi aprovado pelo Comitê de Ética em Pesquisa, conforme parecer (CAEE: 51546215.4.0000.0106). Os participantes incluídos no estudo deveriam estar regularmente matriculados na faculdade selecionada para a pesquisa, ter idade entre 18 e 46 anos e apresentar o TCLE devidamente assinado. Foram excluídos do estudo indivíduos com idade inferior a informada, que não apresentaram o TCLE assinado, que não compareceram nos dias marcados para coleta de dados e indivíduos em tratamento para distúrbio do sono. Vale ressaltar que todos os acadêmicos avaliados realizavam outras atividades laborais no período diurno e desempenhavam suas atividades acadêmicas no período noturno.

Foram coletados os dados de massa corporal (MC), estatura (E) e circunferência da cintura (CC). Através desses dados foi calculado o índice de massa corporal (IMC) e a razão cintura para a estatura (RCE). O IMC foi obtido através da divisão da massa corporal $(\mathrm{kg})$ pela estatura $(\mathrm{m})$ elevada ao quadrado $\left(\mathrm{m}^{2}\right)^{9}$. A RCE se deu pela divisão da CC pela $\mathrm{E}$ em $\mathrm{cm}^{10}$. Na classificação do IMC foram utilizados os pontos de corte sugeridos pela Organização Mundial da Saúde $(\mathrm{OMS})^{11}$. A razão cintura para estatura foi classificada de acordo com os pontos de corte 0,5 sugeridos por Corrêa et al. ${ }^{12}$.

Sendo assim, a massa corporal foi mensurada com uma balança antropométrica (Welmy ${ }^{\circledR}$, modelo 110FF, São Paulo, Brasil), com precisão de $100 \mathrm{~g}$, na qual os sujeitos foram avaliados em pé, descalços e vestindo roupas leves. Duas medidas foram realizadas, obtendo-se a média aritmética entre elas e em caso de diferença acima de $200 \mathrm{~g}$, foi realizada nova mensuração ${ }^{13}$.

A estatura foi obtida por meio de estadiômetro portátil da marca $W_{I S O}{ }^{\circledR}$, afixado a parede, graduado em centímetros e milímetros, com os sujeitos descalços e posicionados em pé sobre a base do estadiômetro. A avaliação foi feita com o avaliado em apneia inspiratória, obedecendo ao plano de Frankfurt. Duas medidas foram realizadas, obtendo-se a média aritmética entre elas e em caso de diferença superior a $0,2 \mathrm{~cm}$ entre as medidas foi realizada uma nova medição ${ }^{13}$.

Para a circunferência da cintura (CC), os avaliados foram colocados em posição ereta, com o mínimo de roupa possível, efetuando a medida com fita métrica inelástica na distância média entre a última costela flutuante e a crista ilíaca ${ }^{14}$. Utilizaram-se os pontos de corte definidos por Lean et al. ${ }^{15}$ considerando-se adequada ou normal $\mathrm{CC}<80 \mathrm{~cm}$ para mulheres e $<94 \mathrm{~cm}$ para homens. 
A pressão arterial (PA) foi mensurada com um esfigmomanômetro da marca (Solidor ${ }^{\circledR}$, Brasil) de coluna de mercúrio, com o auxílio de um estetoscópio da marca Rappaport (Premium ${ }^{\circledR}$, Brasil), a pressão arterial sistólica (PAS) e pressão arterial diastólica (PAD) foram aferidas no braço direito do sujeito, postado ao nível do coração. A PA foi determinada de acordo com a padronização proposta pela $7^{\circ}$ Diretriz Brasileira de Hipertensão Arterial ${ }^{16}$. A PAS foi assinalada na 1a fase de Korotkoff (aparecimento do ruído), e a PAD, na $5^{\underline{a}}$ fase de Korotkoff (desaparecimento do ruído). Utilizaram-se como pontos de corte para PA aumentada valores de PAS $\geq 140 \mathrm{mmHg}$ e/ou PAD $\geq 100 \mathrm{mmHg}^{16}$. Foram realizadas três mensurações da PA. A primeira mensuração foi realizada após o indivíduo permanecer sentado em repouso por um período mínimo de cinco minutos em um local calmo. Já a segunda mensuração foi realizada após 3 minutos da primeira e o mesmo foi realizado para a terceira avaliação. Para chegar a PA final de cada indivíduo foi realizada a média aritmética das três mensurações.

A qualidade do sono foi analisada com base no instrumento denominado Índice de Qualidade do Sono de Pittsburgh (PSQI). A versão adotada foi anteriormente traduzida, validada e ajustada conforme os padrões culturais brasileiros. O PSQI avalia a qualidade do sono no último mês e é composto por dez questões que contemplam sete componentes: a qualidade subjetiva do sono; a latência do sono; a duração do sono; a eficiência habitual do sono; os distúrbios do sono; o uso de medicação para dormir; e a sonolência diurna e distúrbios durante o dia. O referido instrumento possui uma pontuação máxima de 21 pontos. Os universitários com escores superiores a cinco pontos foram classificados como maus dormidores ${ }^{8}$. A qualidade do sono foi classificada de acordo com a National Sleep Foundation's ${ }^{17}$.

Para a análise dos dados foi realizada a estatística descritiva composta por média, desvio padrão e frequência. Foi utilizado o teste de Shapiro-Wilk para verificar a normalidade dos dados. Para verificar as diferenças dos gêneros entre as proporções do estado nutricional, PAS, PAD e qualidade do sono foram realizados os testes de Qui-quadrado e Exato de Fisher. Também foi realizada regressão binária logística com um nível significância de $p<0,05$ e intervalo de confiança de $95 \%$. Todas as análises foram realizadas no software SPSS versão 20.0 .

\section{RESULTADOS}

A população total desse estudo foi construída por 250 acadêmicos. Destes, 48 foram excluídos, sendo, 8 com idade inferior a informada, 29 que não apresentaram o TCLE assinado, 10 que não compareceram nos dias marcados para coleta de dados e 1 (um) indivíduo em tratamento de distúrbio do sono. Sendo assim, o presente estudo foi composto por 202 acadêmicos (126 homens e 76 mulheres), com idades entre 18 e 46 anos.

A Tabela 1 apresenta os dados para caracterização da amostra. Dessa forma, não foram encontradas diferenças entre as variáveis mensuradas. Podemos destacar que as variáveis de IMC, CC, RCE, PAS, PAD e duração do sono estão de acordo com os pontos de corte sugeridos pela literatura.

Tabela 1. Descrição da amostra de universitários diferenciados pelo sexo.

\begin{tabular}{lcc}
\hline Variáveis & $\begin{array}{c}\text { Homens } \\
(\mathbf{n = 1 2 6})\end{array}$ & $\begin{array}{c}\text { Mulheres } \\
(\mathbf{n}=\mathbf{7 6})\end{array}$ \\
Idade (anos) & $24,69 \pm 6,37$ & $22,77 \pm 4,95$ \\
Massa Corporal $(\mathrm{kg})$ & $75,83 \pm 13,83$ & $60,85 \pm 11,24$ \\
Estatura $(\mathrm{cm})$ & $175,63 \pm 6,93$ & $163,19 \pm 7,27$ \\
IMC $\left(\mathrm{kg} / \mathrm{m}^{2}\right)$ & $24,50 \pm 3,64$ & $22,81 \pm 3,53$ \\
Circunferência da cintura $(\mathrm{cm})$ & $84,84 \pm 10,34$ & $79,90 \pm 9,13$ \\
RCE $(\mathrm{cm})$ & $0,48 \pm 0,05$ & $0,49 \pm 0,05$ \\
PAS $(\mathrm{mm} / \mathrm{Hg})$ & $119,04 \pm 13,53$ & $111,55 \pm 10,64$ \\
PAD $(\mathrm{mm} / \mathrm{Hg})$ & $74,84 \pm 12,50$ & $71,42 \pm 8,69$ \\
Qualidade do Sono $(\mathrm{h})$ & $7,1 \pm 1,4$ & $7,6 \pm 1,4$ \\
\hline
\end{tabular}

IMC: índice de massa corporal; RCE: relação cintura estatura; PAS: pressão arteria sistólica; PAD: pressão diastólica.

A Tabela 2 apresenta as comparações das proporções obtidas no IMC, CC, RCE, PAS, PAD e qualidade do sono entre os gêneros. Não foi possível observar diferenças significativas nas variáveis mensuradas. Podemos destacar que grande maioria dos universitários masculinos avaliados apresentou IMC (58,7\%), CC (90,5\%), RCE (68,3\%), PAS $(90,5 \%)$, PAD $(95,2 \%)$ e qualidade do sono $(67,5 \%)$ classificado como normal. Em relação às universitárias, grande maioria apresentou IMC (75,0\%), CC (85,5\%), RCE $(57,9 \%)$, PAS $(97,4 \%)$ e PAD $(100 \%)$ e qualidade do sono $(71,1 \%)$ classificado como normal.

A Tabela 3 apresenta valores sobre as variáveis contidas na pesquisa em relação ao tempo de sono com os fatores de risco a saúde. No grupo de universitários, foi possível observar que 7,1 horas de sono apresenta proteção e prevenção para o aumento da PAS (OR=0,31; $p=0,04)$. Já nas universitárias constatou que 7,6 horas de sono apresenta associação com a RCE para a proteção e prevenção dos fatores riscos de desenvolvimento de doenças cardiovasculares $(\mathrm{OR}=2,57$; $p=0,03)$. 
Tabela 2. Comparações das proporções do IMC, CC, RCE, PAS, PAD e qualidade do sono entre os gêneros.

\begin{tabular}{|c|c|c|c|}
\hline Variáveis & $\begin{array}{c}\text { Homens } \\
(\%)\end{array}$ & $\begin{array}{l}\text { Mulheres } \\
\text { (\%) }\end{array}$ & Valor de $p$ \\
\hline \multicolumn{4}{|l|}{ IMC $\left(\mathrm{kg} / \mathrm{m}^{2}\right)$} \\
\hline Baixo Peso & $0,8(n=1)$ & $3,9(n=3)$ & \multirow{4}{*}{$0,06^{*}$} \\
\hline Eutrófico & $58,7(n=74)$ & $75,0(n=57)$ & \\
\hline Sobrepeso & $31,0(n=39)$ & $17,1(n=13)$ & \\
\hline Obesidade & $9,5(n=12)$ & $3,9(n=3)$ & \\
\hline \multicolumn{4}{|l|}{$\mathrm{CC}(\mathrm{cm})$} \\
\hline Normal & $90,5(n=114)$ & $85,5(n=65)$ & \multirow{2}{*}{$0,38^{* *}$} \\
\hline Alterado & $9,5(n=12)$ & $14,5(n=11)$ & \\
\hline \multicolumn{4}{|l|}{$\mathrm{RCE}(\mathrm{cm})$} \\
\hline Normal & $68,3(n=86)$ & $57,9(n=44)$ & \multirow{2}{*}{$0,14^{* *}$} \\
\hline Alterado & $31,7(n=40)$ & $42,1(n=32)$ & \\
\hline \multicolumn{4}{|l|}{ PAS $(\mathrm{mm} / \mathrm{Hg})$} \\
\hline Normal & $90,5(n=114)$ & $97,4(n=74)$ & \multirow{2}{*}{$0,06^{*}$} \\
\hline Alterado & $9,5(n=12)$ & $2,6(n=2)$ & \\
\hline \multicolumn{4}{|l|}{$\mathrm{PAD}(\mathrm{mm} / \mathrm{Hg})$} \\
\hline Normal & $95,2(n=120)$ & $100(n=76)$ & \multirow{2}{*}{$0,12^{*}$} \\
\hline Alterado & $4,8(n=6)$ & $0(n=0)$ & \\
\hline \multicolumn{4}{|c|}{ Qualidade do Sono (h) } \\
\hline Normal & $67,5(n=85)$ & $71,1(n=54)$ & \multirow{2}{*}{$0,53^{* *}$} \\
\hline Alterado & $32,5(n=41)$ & $28,9(n=22)$ & \\
\hline
\end{tabular}

* Exato de Fischer; ${ }^{* *}$ Qui-quadrado.

IMC: índice de massa corporal; CC: circunferência da cintura; RCE: relação cintura estatura; PAS: pressão arterial sistólica; PAD: pressão diastólica; $n$ : número de sujeitos; Valor de $p$ : nível de significância.

Tabela 3. Associação do tempo de sono com os fatores de risco a saúde.

\begin{tabular}{|c|c|c|c|c|c|c|}
\hline \multirow{2}{*}{ Variáveis } & \multicolumn{3}{|c|}{$\begin{array}{l}\text { Homens } \\
(n=126)\end{array}$} & \multicolumn{3}{|c|}{$\begin{array}{l}\text { Mulheres } \\
(n=76)\end{array}$} \\
\hline & Odds Ratio & IC (95) & Valor de $p$ & Odds Ratio & IC (95) & Valor de $p$ \\
\hline \multicolumn{7}{|l|}{ Antropométricas } \\
\hline IMC (kg/m²) & 0,89 & $(0,58-1,46)$ & 0,23 & 1,11 & $(0,50-2,49)$ & 0,39 \\
\hline $\mathrm{CC}(\mathrm{cm})$ & 1,72 & $(0,37-8,15)$ & 0,15 & 0,84 & $(0,13-5,46)$ & 0,56 \\
\hline RCE (cm) & 1,00 & $(0,30-3,36)$ & 0,44 & 2,57 & $(1,68-9,77)$ & $0,03^{*}$ \\
\hline \multicolumn{7}{|l|}{ Cardiovasculares } \\
\hline PAS (mm/Hg) & 0,31 & $(1,06-1,77)$ & $0,04 *$ & 1,76 & $(0,19-9,99)$ & 0,11 \\
\hline PAD $(\mathrm{mm} / \mathrm{Hg})$ & 3,85 & $(0,39-39,91)$ & 0,32 & 0,25 & $(1,08-3,02)$ & 0,31 \\
\hline
\end{tabular}

Estatística Odds Ratio: Regressão Binária Logística.

IMC: índice de massa corporal; CC: circunferência da cintura; RCE: relação cintura estatura; PAS: pressão arterial sistólica; PAD: pressão diastólica; IC: intervalo de confiança.

* Nível de significância.

\section{DISCUSSÃO}

O presente estudo teve como objetivo avaliar a associação entre o tempo de sono com os fatores de risco antropométricos e cardiovasculares em universitários. Diante disso, foi observado que o IMC não apresentou associação com o tempo de sono, tanto no grupo de homens, quanto no grupo de mulheres. O mesmo ocorreu nas comparações das classificações obtidas. O IMC apresentou média de
$24,50 \pm 3,54 \mathrm{~kg} / \mathrm{m}^{2}$ para os homens, onde foi observado que $31 \%$ apresentaram sobrepeso e $9,5 \%$ encontram-se com obesidade. Já média das mulheres foi de $22,81 \pm 3,53 \mathrm{~kg} / \mathrm{m}^{2}$, onde os dados do IMC revelam que 17,1\% estão classificadas como sobrepeso e 3,9\% se encontram com na classificação de obesidade.

Com relação a quantidade de sono foi observado que os homens dormem em média 7,1 $\pm 1,4$ e as mulheres $7,6 \pm 1,4$, horas respectivamente. Sendo assim, ambos os sexos apre- 
sentaram sono adequado, conforme as recomendações do National Sleep Foundation ${ }^{17}$.

Atualmente, estudos têm apontado que a má qualidade do sono tem contribuído diretamente para a hiperfagia, intolerância à glicose e perda de massa magra ${ }^{18}$, além de estar relacionado a um aumento significativo da adiposidade corporal $^{19}$ elevando os valores do $\mathrm{IMC}^{20}$. Souza et al. ${ }^{21}$ destacam que menos de seis horas de sono por noite estão relacionados com o aumento do IMC e consequentemente do sobrepeso e obesidade, pois estes mesmos autores enfatizam que a privação do sono associa-se significativamente com excesso de peso, onde o risco de se ter sobrepeso e obesidade aumenta em 2,3 vezes quando comparados com aqueles que apresentam uma boa qualidade do sono. No presente estudo foi observado que o risco relativo entre a qualidade de sono e o IMC foi de 0,89 para os homens e 1,11 para as mulheres, no entanto estes valores não representaram associação significativa.

Cabe mencionar que apesar do IMC ser considerado um bom indicador de risco, os seus valores devem ser tratados com cautela, pois uma das suas limitações é a de não distinguir o tecido adiposo da massa livre de gordura como é o caso do tecido musculares esquelético, podendo superestimar o nível de adiposidade em um indivíduo musculoso, por exemplo, e classificá-lo com risco a saúde ${ }^{22}$.

Quanto a CC, foi observada uma média de $84,84 \pm$ $10,34 \mathrm{~cm}$ para os homens e $78,90 \pm 9,13 \mathrm{~cm}$ para as mulheres, faixa considerada normal e sem complicações metabólicas, porém também foi constatado que 9,5\% dos homens e $14,5 \%$ das mulheres apresentavam CC alterada. Contudo, esses dados não foram significativos nas proporções e não apresentou associação com a qualidade do sono.

Vasconcelos et al. ${ }^{23}$ sustentam que os universitários maus dormidores apresentam valores de IMC e CC superiores aos bons dormidores, onde os distúrbios do sono apresentam correlação significativa com os indicadores antropométricos, dentre eles a CC, no entanto tal fato também não foi observado no presente estudo. Ford et al. ${ }^{24}$ afirmam que a duração do sono está associada com anormalidades antropométricas. Os resultados de seu estudo acrescentam que a duração do sono foi associada ao IMC e CC de maneira linear inversa. Ainda com relação a intenção do presente estudo em associar indicadores antropométricos com a duração do sono, a CC quando alterada se constitui como um forte preditor de hipertensão arterial, sendo a obesidade central considerada pela comunidade acadêmica como um importante fator de risco cardiovascular e metabólico ${ }^{25}$.

Outro indicador antropométrico mensurado no presente estudo foi a RCE onde os valores médios de 0,48 $\pm 0,05$ para homens e $0,49 \pm 0,05$ para as mulheres, centímetros respectivamente. Assim sendo, 31,7\% dos homens e 42,1\% das mulheres apresentaram RCE alterada, porém estes valores não resultaram em diferenças significativas nas comparações das proporções.

Já na associação da RCE com o tempo de sono foi possível observar que existiu um fator protetor dos fatores risco de desenvolvimento de doenças cardiovasculares para o grupo de mulheres $(\mathrm{OR}=2,57 ; p=0,03)$ pois as mesmas apresentaram uma média de 7,6 horas de sono, dado este que se enquadra nas recomendações diárias de sono para a manutenção e restauração das funções orgânicas, podendo explicar essa associação positiva ${ }^{17}$. É importante destacar que para este estudo a média da RCE encontra-se dentro dos pontos de corte de normalidade ${ }^{15}$ para $57,9 \%$ das mulheres avaliadas, característica importante, haja vista que a RCE é um preditor para doenças cardiovasculares e riscos metabólicos ${ }^{26}$. A duração do sono e as medidas antropométricas ainda necessita de atenção pela literatura científica ${ }^{24}$, havendo demanda para novas publicações que esclareçam a real influência destes marcadores de saúde na quantidade e qualidade do sono $^{23}$.

A literatura apresenta ainda dados que merecem atenção como é o caso da pressão arterial ${ }^{27}$, onde os universitários do sexo masculino que obtiveram valores de tempo de sono médio em 7,1 horas, segundo os dados mensurados no presente estudo, estando protegidos da pressão arterial elevada. Com relação a pressão arterial sistólica e diastólica, os dados do presente estudo corroboram com a literatura onde os valores médios de PAS dos indivíduos do sexo masculino foi de PAS $=119,04 \pm 13,53 \mathrm{mmHg}$ e no sexo feminino de $P A S=111,5 \pm 10,64 \mathrm{mmHg}$, enquanto as médias da PAD foram de $\mathrm{PAD}=74,84 \pm 12,50 \mathrm{mmHg}$ para homens e $\mathrm{PAD}=72,8 \pm 8,69 \mathrm{mmHg}$ para mulheres. Contudo, não podemos deixar de mencionar que a média da PAS e PAD está dentro da normalidade, entretanto estes achados limitam-se apenas a uma medida, fazendo com que os resultados encontrados de associação da PA com a qualidade do sono sejam vistos com cautela, devido à baixa relevância clinica quando são coletados dados de PA apenas uma vez ${ }^{27}$, ficando vulnerável a consideração deste parâmetro como um fator de risco ${ }^{28}$.

Rocha e Martino ${ }^{29}$ sugerem que dormir menos de seis horas por noite pode estar ligado ao aumento da PA e com o risco de desenvolver hipertensão arterial sistêmica. Outro distúrbio a ser considerado é a apneia do sono que pode contribuir para o aparecimento de problemas $\operatorname{cardía} \cos ^{30}$. Martins et al. ${ }^{31}$ destacam que os indivíduos do sexo masculino são mais suscetíveis a hipertensão arterial sistêmica, deixando a população jovem adulta em alerta para os fatores de risco que contribuem direta e indiretamente para a elevação 
da PA, como é o caso do acúmulo de gordura corporal na região abdominal, alimentação inadequada com o consumo de sódio acima dos valores nutricionais recomendados e sedentarismo.

A privação do sono esta interligada com os riscos de fatores antropométricos e cardiovasculares em universitários ${ }^{8}$, além de contribuir para a redução na eficiência do processamento cognitivo, diminui o tempo de reação e a responsividade atencional, incluindo prejuízo na memória, aumento da irritabilidade, alterações endócrinas, imunológicas, cansaço, náuseas, dores de cabeça, ardência nos olhos, visão turva, dores articulares e diminuição da libido ${ }^{32}$.

Segundo Bernardo et al. ${ }^{33}$ devido às suas consequências, os distúrbios do sono têm sido considerados um problema de saúde pública que necessita ser cuidadosamente avaliado e enfrentado com diversas estratégias. Já que o período universitário muitas vezes altera a rotina diária, poderia ser considerado por parte das universidades intervenções para a conscientização sobre a importância do sono com o intuito de aperfeiçoar a qualidade de vida da população acadêmica.

Vale ressaltar que o presente estudo contém as seguintes limitações: não utilizou parâmetros sanguíneos e plasmáticos como indicativo de risco à saúde, não foi realizada uma análise de composição corporal e não foi utilizada nenhuma tecnologia padrão para a análise da duração do sono. Do ponto de vista clinico apenas uma mensuração da PA não pode se configurar adequadamente como fator de risco. Tais variáveis devem ser controladas em futuras pesquisas sobre a temática.

Podemos concluir que uma média de 7,1 horas de sono contribui para a prevenção e manutenção da PAS em universitários do sexo masculino. Enquanto isso, no sexo feminino, uma média 7,6 horas de sono contribuem na prevenção e proteção da RCE elevada e do risco de desenvolvimento de doenças cardiovasculares.

\section{REFERÊNCIAS}

1. Barbosa KTF, Oliveira FMRL, Oliveira SM, Gomes MO, Fernandes MGM. Qualidade do sono em pacientes idosos em atendimento ambulatorial. Rev Enferm UFPE. 2016;10(Supl. 2):756-61. https:// dx.doi.org/10.5205/reuol.6884-59404-2-SM-1.1002sup201609

2. Oliveira AKSM, Santos OCB, Cruz GPS, Correia LNAL, Correia KVD. Avaliação da qualidade do sono de pacientes em tratamento ortodôntico. Rev Virtual AcBO. 2016;25(2):226-31.

3. Fonseca ALP, Zeni LB, Flügel NY, Sakae TM, Remor KVT. Estudo comparativo sobre qualidade do sono entre universitários de uma instituição de ensino do sul catarinense. Arq Catarin Med. 2015;44(4):21-33.

4. Silva GM, Ramos FA, Bernandes LS, Alves PRR, Fabro MA. Qualidade do sono em estudantes do regime regular e internato médico. Rev Med UFPR. 2016;3(1):19-24. https://doi.org/10.5380/ rmu.v3i1.44136
5. Batista M, Souza WCS, Lima VA, Grzelczak MT, Mascarenhas LPG Qualidade do sono de adolescentes da área rural e a associação com o estado nutricional. Interfaces Cient Saúde Amb. 2017;5(2):916. https://doi.org/10.17564/2316-3798.2017v5n2p9-16

6. Costa FF, Freitas AG, Jesus LG, Magalhães FMC, Pereira CCR, Silva GMG, Tormin KRA, Costa SM, Macedo SBM, Ramos MTO. Qualidade de sono e estresse em universitários dos últimos semestres dos cursos da área da saúde. E-RAC. 2015;5(1):1-15.

7. Araújo DF, Almondes KM. Qualidade de sono e sua relação com o rendimento acadêmico em estudantes universitários de turnos distintos. Psico. 2012;43(3):350-9.

8. Araújo MFM, Lima ACS, Alencar AMPG, Araújo TM, Fragoaso LVC, Damasceno NNC. Avaliação da qualidade do sono de estudantes universitários de Fortaleza-CE. Texto Contexto Enferm. 2013;22(2): 352-60. https://doi.org/10.1590/S0104-07072013000200011

9. Leite SP, Gomes LPS, Lemos CFS, Lessa KMR, Pedral RL, Brandão PP. Métodos de obtenção de dados antropométricos confiáveis. Ciênc Biol Saúde. 2015;3(1):87-100.

10. Moraes KD, Araújo AP, Santos AF, Barbosa JMA, Martins MLB. Correlação entre o índice de massa corporal e indicadores antropométricos de risco cardiovascular em mulheres. Rev Pesq Saúde. 2015;16(3):175-81

11. World Health Organization. Obesity: preventing and managing the global epidemic. Report of a World Health Organization Consultation. Geneva: WHO, 2000. p. 256. WHO Obesity Technical Report Series, n. 284

12. Corrêa MM, Tamasi S, Thumé E, Oliveira ERA, Fachini LA. Razão cintura-estatura como marcador antropométrico de excesso de peso em idosos brasileiros. Cad Saúde Pública. 2017;33(5):e00195315. https://doi.org/10.1590/0102-311X00195315

13. Smolarek AC, Bernard BRB, Bueno JCA, Mascarenhas LPG, Souza Junior TP. Associação do perfil glicêmico com estado nutricional e pressão arterial sistêmica de adolescentes. Arq Ciênc Saúde. 2015;22(4):31-5. https://doi.org/10.17696/23183691.22.4.2015.69

14. Leone C, Nascimento VG, Silva JPC, Bertoli CJ. Razão cintura/estatura: Marcador de alteração nutricional em pré-escolares. J Hum Growth Dev. 2014;24(3):289-94. https://doi.org/10.7322/jhdg.88962

15. Lean ME, Han TS, Morrison CE. Waist circumference as a measure for indicating need for weight management. Br Med J. 1996;311(15):158-61.

16. Sociedade Brasileira de Cardiologia. 7o Diretriz Brasileira de Hipertensão Arterial. Arq Bras Cardiol. 2016;107(3 supl.3):1-83. https://doi.org/10.5935/abc.20160140

17. Hirshkowitz M, Whiton K, Albert SM, Alessi C, Bruni O, DonCarlos L, Hazen N, Herman J, Katz ES, Kheirandish-Gozal L, Neubauer DN, O'Donnell AE, Ohayon M, Peever J, Rawding R, Sachdeva RC, Setters B, Vitielo MV, Ware JC, Hillard PJA. National Sleep Foundation's sleep time duration recommendations: methodology and results summary. Sleep Health. 2015;1(1):40-3. https://doi. org/10.1016/j.sleh.2014.12.010

18. Araújo MFM, Freitas RWJF, Lima ACS, Pereira DCR, Zanetti ML, Damasceno MMC. Indicadores de saúde associados com a má qualidade do sono de universitários. Rev Esc Enferm USP. 2014;48(6):1085-92. https://doi.org/10.1590/S0080623420140000700017 
19. Amorim JR, Silva IA, Shimizu IS. Avaliação da qualidade de sono em pacientes com câncer de mama em quimioterapia. Rev Bras Mastologia. 2017;27(1):3-7. https://doi.org/10.5327/ Z201700010002RBM

20. Crispim CA, Zalcman I, Dáttilo M, Padilha HG, Tufik S, Mello MT. Relação entre sono e obesidade: uma revisão da literatura. Arq Bras Endocrinol Metab. 2007;51(7):1041-9. https://doi.org/10.1590/ S0004-27302007000700004

21. Souza FN, Santo ÍlL, Moraes SR, Silva CMS, Ellinger VCM. Associação entre qualidade do sono e excesso de peso entre estudantes de medicina da Universidade Severino Sombra, Vassouras-RJ. Rev Saúde. 2017;8(S1):99-100.

22. Deurenberg P, Deurenberg MY, Wang J, Lin FP, Schmidt G. The impact of body build on the relationship between body mass index and percent body fat Int J Obes Relat Metab Disord. 1999;23(5): 537-42. https://doi.org/10.1038/sj.ijo.0800868

23. Vasconcelos HCA, Fragoso LVC, Marinho NBP, Araújo MFM, Freitas RWJF, Zanetti ML, Damasceno MMC. Correlação entre indicadores antropométricos e a qualidade do sono de universitários brasileiros. Rev Esc Enferm USP. 2013;47(4):852-9. https://doi.org/10.1590/ reeusp.v47i4.78034

24. Ford ES, Li C, Wheaton AG, Chapman DP, Perry GS, Croft JB. Sleep duration and body mass index and waist circumference among US adults. Obesity. 2014;22(4):598-607. https://doi.org/10.1002/ oby. 20558

25. Pires CGS, Mussi FC. Excesso de peso em universitários ingressantes e concluintes de um curso de enfermagem. Esc Anna Nery. 2016;20(4):e20160098. https://doi.org/10.5935/14148145.20160098
26. Madruga JG, Silva FM, Adami FS. Associação positiva entre razão cintura-estatura e presença de hipertensão em adolescentes. Rev Port Cardiol. 2016;35(9):479-84. https://doi.org/10.1016/j. repc.2016.03.004

27. Tufik JM, Geleilete EBC, FN. Medida residencial da pressão arterial. Rev Bras Hipertens. 2009;16(3):186-9.

28. Klein $\mathrm{CH}$, Coutinho ESF, Camacho LAB. Variação da pressão arterial em trabalhadores de uma siderúrgica. Cad Saúde Pública. 1986; 2(2):212-26. https://doi.org/10.1590/S0102-311X1986000200008

29. Rocha MCP, Martino MMF. Estresse e qualidade do sono entre enfermeiros que utilizam medicamentos para dormir. Acta Paul Enferm. 2009;22(5):658-65. https://doi.org/10.1590/ S010321002009000500010

30. Drager LF, Ladeira RT, Brandão-Neto RA, Lorenzi-Filho G, Benseñor IM. Síndrome da apnéia obstrutiva do sono e sua relação com a hipertensão arterial sistêmica. Evidências atuais. Arq Bras Cardiol. 2002;78(5):531-6. https://doi.org/10.1590/S0066-782X 2002000500013

31. Martins MCC, Ricarte IF, Rocha CHL, Maia RB, Silva VB, Veras $A B$, Souza Filho MD. Pressão arterial, excesso de peso e nível de atividade física em estudantes de universidade pública. Arq Bras Cardiol. 2010;95(2):192-9. https://doi.org/10.1590/S0066782X2010005000069

32. Neves GSML, Giorelli AS, Florido P, Gomes MM. Transtornos do sono: visão geral. Rev Bras Neurol. 2013;49(2):57-71.

33. Bernardo VM, Silva FC, Ferreira EG, Bento GG, Zilch MC, Sousa A, Silva R. Atividade física e qualidade de sono em policiais militares. Rev Bras Ciênc Esporte. 2018;40(2):131-7. https://doi. org/10.1016/j.rbce.2018.01.011 